\title{
The identification of established modifiable mid-life risk factors for cardiovascular disease which contribute to cognitive decline: Korean Longitudinal Study of Aging (KLoSA)
}

\author{
Yebeen Ysabelle Boo ${ }^{1,2,3}$ (D) $\cdot$ Otto-Emil Jutila ${ }^{1}$ (D) $\cdot$ Meghan A. Cupp ${ }^{3,4,5}$ (D) $\cdot$ Logan Manikam $^{3,4}$ (D) $\cdot$ Sung-II Cho ${ }^{6}$ (D)
}

Received: 24 July 2020 / Accepted: 25 December 2020 / Published online: 4 February 2021

(c) The Author(s) 2021

\begin{abstract}
Introduction We explored how different chronic diseases, risk factors, and protective factors highly associated with cardiovascular diseases (CVD) are associated with dementia or Mild Cognitive Impairment (MCI) in Korean elders, with a focus on those that manifest in mid-life.

Methods A CVD-free cohort ( $n=4289$ ) from the Korean Longitudinal Study of Aging was selected to perform Cox mixedeffects proportional hazard regressions. Eighteen control variables with strong associations to CVD were chosen as explanatory variables, and Mini-Mental State Examination (MMSE) score cut-off for dementia and MCI were used as outcome variables.

Results The statistically significant $(P<0.05)$ adverse factors that contribute in developing dementia were age (aHR 1.07, 1.05-1.09), Centre for Epidemiological Studies Depression Scale (CESD-10) (aHR 1.17, 1.12-1.23), diagnosis with cerebrovascular disease (aHR 3.73, 1.81-7.66), living with diabetes (aHR 2.30, 1.22-4.35), and living with high blood pressure (HBP) (aHR 2.05, 1.09-3.87). In contrast, the statistically significant protective factors against developing dementia were current alcohol consumption (aHR 0.67, 0.46-0.99), higher educational attainment (aHR 0.36, 0.26-0.56), and regular exercise (aHR $0.37,0.26-0.51$ ). The factors with a statistically significant adverse association with progression to MCI were age (aHR 1.02, 1.01-1.03) and CESD-10 (aHR 1.17, 1.14-1.19). In contrast, the statistically significant protective factors against developing MCI were BMI (aHR 0.96, 0.94-0.98), higher educational attainment (aHR 0.33, 0.26-0.43), and regular exercise (aHR 0.83, 0.74-0.92).

Conclusion In lieu of the protective factor of MCI and dementia, implementing regular exercise routine well before mid-life and cognitive decline is significant, with adjustments made for those suffering from health conditions, so they can continue exercising despite their morbidity. Further attention in diabetes care and management is needed for patients who already show decline in cognitive ability as it is likely that their MCI impacts their ability to manage their existing chronic conditions, which may adversely affect their cognitive ability furthermore.
\end{abstract}

Keywords Dementia $\cdot$ Mild cognitive impairment $\cdot$ Cardiovascular diseases $\cdot$ Korean longitudinal study of aging · Epidemiology

Yebeen Ysabelle Boo

yebeen.boo@dph.ox.ac.uk

1 Nuffield Department of Population Health, University of Oxford, Oxford, UK

2 Population, Policy and Practice, UCL Great Ormond Street Institute of Child Health, London, UK

3 Department of Epidemiology and Public Health, UCL Institute of Epidemiology and Health Care, London, UK
4 Aceso Global Health Consultants Ltd, London, UK

5 Brown University School of Public Health, Providence, Rhode Island, USA

6 Graduate School of Public Health and Institute of Health and Environment, Seoul National University, Seoul, Republic of Korea 


\section{Introduction}

The 2016 Global Health Observatory data indicates that four of the top five causes of mortality worldwide are non-communicable diseases (NCDs) [1]. These NCDs include ischaemic heart disease, stroke, chronic obstructive pulmonary disease (COPD), and Alzheimer's disease (AD) and other dementias. All these high burden NCDs are either a type of cardiovascular disease (CVD) or are closely associated with CVDs. This is indicative of the fact that CVD is the number one cause of mortality globally, with the World Health Organisation estimating mortality due to CVD at 17.9 million cases every year [2]. CVDs are a group of heart and blood vessel disorders, including coronary heart disease (CHD), cerebrovascular disease, and stroke. CVDs are an important comorbidity of COPD, as lower oxygen levels due to COPD may put additional strain on the heart and can contribute to heart failure [3]. Some well-understood non-modifiable risk factors for CVDs are age, ethnic background, and family history of CVDs. Modifiable risk factors include smoking, high blood pressure, diabetes, physical inactivity, being overweight or obese, and having high blood cholesterol [4]. Stress is also considered a risk factor for CVD, as high stress levels can either contribute to or be a consequence of a higher blood pressure, or an unhealthy lifestyle, such as habitual smoking, and increased alcohol consumption. There is also risk of reverse causality between socio-economic factors and health [5-7]. Socio-economic position is associated with an increased risk of developing NCDs, due to the social gradient in health and their life course epidemiology [8-10].

The mortality due to AD and other dementias, which more than doubled between 2000 and 2006, is one of the biggest challenges faced by the aging population [11]. It poses a major global public health crisis with great social, health, and economic costs, and the number of cases is predicted to double every 20 years [12]. The full extent of support and resources required by dementia patients is not captured, such as the impact on the quality of life for the caregiver and dementia patient. Dementia is an umbrella term to describe a group of neurodegenerative diseases which cause progressive and irreversible deterioration in cognitive function. Cognitive decline occurs in one or more cognitive domains: memory, emotion, behaviour, personality, visuospatial skills, and language [13]. The cognitive decline in dementia cases is severe enough to prevent the individual from functioning independently in daily or social activities [14]. There are several forms of dementia, including AD, vascular dementia, dementia with Lewy bodies, and Parkinson's disease dementia. It is possible to simultaneously present with two or more forms of dementia, further complicating the classification and diagnosis of disease at autopsy [15]. Mild cognitive impairment (MCI) is often one of the first observable signs in the development of dementia, making it an important prognostic marker [16]. Patients with MCI have a greater cognitive decline compared to controls [17]. MCI primarily differs from dementia, since it is not severe enough to prevent the individual from undertaking complex activities [18]. Currently, it is estimated that between 10 and 20\% of the world's elderly population (aged 65 and above) is affected by MCI [19] and, although not all MCI patients will progress to further cognitive impairment or dementia, many MCI patients are identified to have a very mild form of dementia [20].

To date, there have been several longitudinal studies investigating the associations between cardiovascular risk factors and dementia [21-23]. Dementia, unlike other major chronic non-communicable diseases, has only symptomatic treatment; therefore, prevention by early detection and intervention is currently proposed to be the most effective approach for dementia and cognitive impairment [24-28]. Evidence from systematic reviews suggests that more than a third of all dementia cases could be prevented by addressing modifiable risk factors, with CVD (e.g., high blood pressure, HBP) and CVD-related risk factors (e.g., smoking) being significant influences [29]. Observing these risks that manifest in mid-life is particularly significant, as it is a vital point when exposure to risk factors may distinguish between normal aging and the development of dementia [30]. Interventions targeted at the appropriate modifiable risk factors during mid-life may be the most effective in preventing $\mathrm{AD}$; however, this exact process and aetiological element remains unknown [13, 31].

This study focuses on the Republic of Korea (South Korea) population using Korean Longitudinal Study of Aging (KLoSA) data. Associations between modifiable CVD mid-life risk factors and cognitive decline have not been explored in great depth through KLoSA, despite South Korea having one of the most rapidly aging populations and a greater prevalence of dementia than other Asian and Western countries [32]. In fact, these Korean population studies investigating cognition function often focus on a singular factor [33-35]. To further explore risk for cognitive decline and dementia, this study utilises data from five waves of the KLoSA to explore how different chronic diseases and risk factors affect the Mini-Mental State Exam (MMSE) score from 2006 to 2016. This study is distinct from prior work, since the Korean population is rarely a main ethnic group in other well-established longitudinal studies of modifiable risk factors and cognitive health from western populations. This is significant since, even with increasing westernisation, Koreans may have specific environmental, social, lifestyle, and 
genetic factors, which contribute to dementia and CVD risk [36-40]. In particular, Asian individuals experience greater risk of diabetes and HBP at a lower BMI, compared to White counterparts [41] and have a high prevalence of novel genetic variations related to salt sensitivity which can increase the risk of HBP and other CVD events [42, 43]. For example, Koreans have a lower cut-off points for BMI-defined obesity, compared to White individuals, due to having an elevated body fat levels at the same BMI and increased risk of obesity-related cardiometabolic diseases [56]. Therefore, to the best of our knowledge, this is the first completed longitudinal countrywide study involving a middle-age population-based cohort study for investigating the association of CVD, CVD risk factors, and lifestyle factors, with all-cause MCI and dementia in a Korean population. This study, which utilises data from an under-researched ethnic group, will allow for the comparison of associations found from literature using international data and will add to existing knowledge about targeted health interventions to improve the health and well-being of aging populations.

\section{Methods}

The KLoSA dataset includes a random sample of 10,254 adults born before 1961, who are 45 or older at the time of data collection and reside in Korea (excluding Jeju Island). Initiated in 2006, the baseline survey is carried out every two years and six waves are completed as of 2020 . A refreshment sample of 920 individuals born between 1962 and 1963 was added to wave 5 in 2014 as before this, KLoSA only hold a closed cohort. This study only utilises data collected from participants of wave 2 and subsequent waves, as institutionalised individuals (e.g., those in assisting living) were initially excluded from wave 1 . More information about KLoSA design can be found in the Division of Behavioral and Social Research (DBSR) International Studies Behavioral and Social Studies DBSR Korean Longitudinal Study of Aging website [44]. Figure 1 shows the criteria which were applied to exclude participants from the original dataset of wave 2 . The final baseline dataset used for data analysis contained 4,289 participants. The participants previously diagnosed with CHD and cerebrovascular diseases were excluded to allow for the assessment of how the development of new chronic diseases or existing lifestyle risk factors affect cognitive decline over time. Twenty-one percent of participants had one or more missing answers. However, out of all variables in the dataset, only nine variables had partial missing data. We excluded participants using list-wise deletion if they

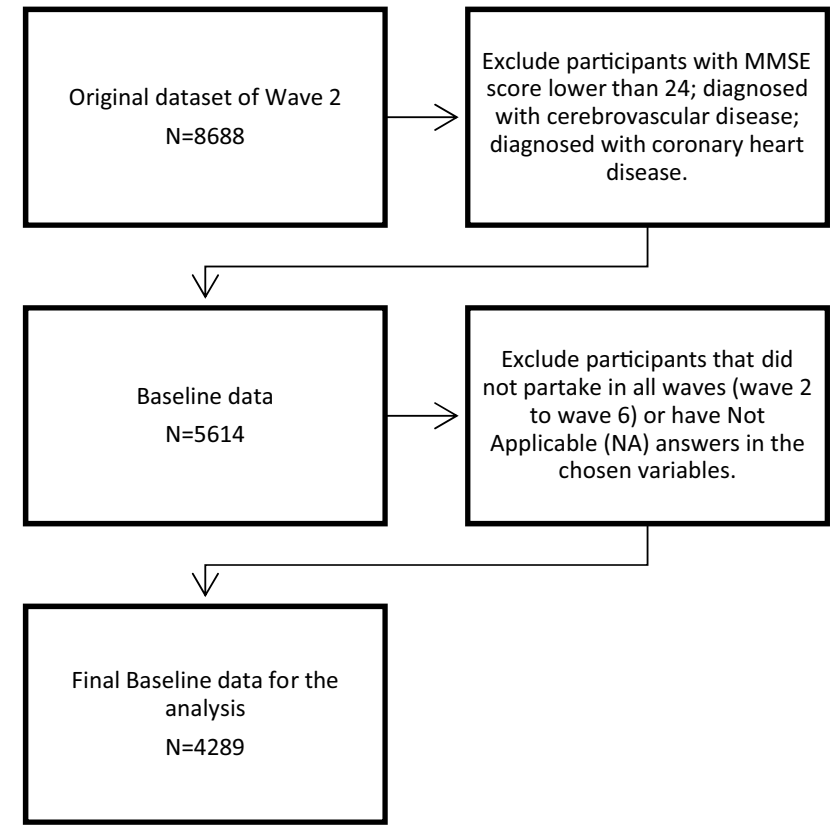

Fig. 1 Flowchart for participant data selection and inclusion and exclusion criteria for study

had not applicable (NA) answers during wave 2 collection point, or if they did not partake in all waves (2-6) [45].

\section{Variable selection}

A total of 16 control variables were chosen to perform Cox mixed-effects proportional hazard regression models with MMSE score as the outcome variable. The MMSE, developed by Folstein in 1975 [46], is a screening test that measures cognitive ability and is often used as a diagnostic tool for MCI. The test is delivered in 7-10 min, and is designed to measure the general cognitive ability of orientation, registration, attention-calculation, recall, and language. It is composed of 30 question scores between 18 and 23 indicate cognitive deficiency and scores 17 and below indicates dementia [47]. The MMSE has a sensitivity of $86.36 \%$ and specificity of $86.36 \%$ for patients who have been educated for five years or more [48]. Multiple measurements of MMSE changes over time can be used to predict the conversion of MCI to dementia [49]. There are few limitations with MMSE itself as a measurement of cognitive skills. It does not account for individual differences; people who have low educational level or in low socio-economic status, they score relatively low due to floor effect (low specificity), whereas with highly educated people, they score relatively high due to celling effect (low sensitivity). 
The following independent variables were chosen to reflect both the physical and mental health of the participants and lifestyle and socio-economic determinates of health:

1. Two mental health components: 10 -item revised Centre for Epidemiological Studies Depression Scale (CESD10); diagnosed with mental health disability;

2. Lifestyle risk factors: Smokes cigarette, drinking habits, Body Mass Index (BMI), regular exercise;

3. Pre-existing conditions: Diagnosed with arthritis or rheumatism; liver diseases; lung diseases; cancer; diabetes; HBP;

4. Newly diagnosed with CVD: cerebrovascular diseases; CHD;

5. Highest year of education.

These independent factors can be sub-grouped as protective factors and risk factors. Protective factors include regular exercise, low CESD-10 score, high satisfaction with economics status, and higher level of education. The risk factors include being diagnosed with various chronic diseases, smoking, and/or drinking habits and high BMI. The biological explanation linking the risk factors of CVD with the risk of cognitive decline provides the rationale of selecting these variables. For example, HBP is a wellknown modifiable CVD risk factor related to impairment in cognitive function and vascular dementia, characterised by alterations in the cerebral vasculature, function, and blood flow [29].

Smoking is another independent risk for CVD, and there is a consensus that it can increase the risk of dementia and cognitive impairment. Smoking is part of multiple pathological pathways for cognitive decline, and is an independent risk factor for stroke, atherosclerosis, inflammatory processes, and oxidative damage to neurons [53]. Moreover, type-II diabetes mellitus (T2DM) is firmly linked with negative alterations to cognitive function, through several domains. Several mechanisms both vascular and non-vascular (hormonal) are proposed to contribute to cognitive decline and the pathophysiological process for T2DM and dementia have a significant overlap [54].

Another example is obesity, which has rapidly increased in the last couple of decades in Korea [55]. A plausible link between obesity and cognition occurs through an indirect effect on CVD risk factors [57]. Finally, while the relationship between alcohol and cognitive decline and dementia has not been firmly established, low-to-moderate alcohol consumption has been suggested to be cardio-protective and even neuroprotective [62], albeit, heavy consumption is neurotoxic and has been associated with a higher risk for cognitive impairment and dementia in later life [63]. However, not many studies have explored the role of ethnicity or
Korean population in alcohol consumption and development of dementia.

In contrast, following three non-modifiable variables were included in the model to control any biological or time-varying factors:

1. Wave of the interview (every two years from 2006 up to 2016)

2. Age

3. Gender

4. Respondent's satisfaction with economic status (as a proxy for subjective wealth due to different living cost being associated with different regions of Korea).

\section{Statistical analysis}

The Chi-squared test and Kruskal-Wallis one-way analysis of variance test were utilised for analysis of categorical and continuous variables, respectively. These analyses conducted to see the initial correlation of each variable against the MMSE score, via descriptive analysis. The univariate Cox proportional hazard regression models were conducted for six modifiable factors and were visualised as graphical plots. The included six modifiable factors were:

1. CESD-10 $(0=$ lowest to $10=$ highest score $)$

2. smoking $(0=$ non-smoker, $1=$ past smoker, $2=$ current smoker)

3. alcohol consumption $(0=$ non-drinker, $1=$ past drinker, $2=$ current drinker)

4. HBP which can also reflect stress, as well as respondent's underlying conditions $(0=$ no, $1=$ yes $)$

5. total number of chronic diseases (sumchron, Min $=0$, $\operatorname{Max}=6$ )

6. regular exercise $(0=$ no, $1=\mathrm{yes})$.

Cox mixed-effects proportional hazard regression models were also used to confirm how each factor influenced the development of dementia (model 1 with MMSE score $\leq 17$ ) and MCI (model 2 with MMSE score 18-23), controlling the random effects of each patient. The rationale behind building two models, with different cut-off points, is to observe and compare which variables accelerate cognitive decline, while the participants show normal cognitive scores relative to the point when they already have a MCI condition (sensitivity analysis). Cox regression models are also useful in assessing survival time, which is appropriate for our data. Model 1 will show which variables accelerate the event of cognitive decline to dementia, while model 2 will show which variables influence the event of cognitive decline up to the development of MCI. The division of two models will allow for the investigation of the effect modifiable risk factors for both MCI and dementia. 
Table 1 Descriptive analysis of the participants $(n=4289)$

\begin{tabular}{|c|c|c|c|c|c|c|c|c|c|}
\hline MMSE score & $24(N=275)$ & $25(N=352)$ & $26(N=466)$ & $27(N=560)$ & $28(N=704)$ & $29(N=835)$ & $\begin{array}{l}30 \\
(N=1097)\end{array}$ & $\begin{array}{l}\text { Total } \\
(N=4289)\end{array}$ & $p$ value \\
\hline \multicolumn{9}{|l|}{ CESD-10 } & $<0.001$ \\
\hline Mean (SD) & $4.07(2.90)$ & $3.53(2.67)$ & $3.50(2.69)$ & $3.33(2.63)$ & $3.00(2.54)$ & $2.58(2.43)$ & $2.34(2.47)$ & $2.96(2.62)$ & \\
\hline $\begin{array}{l}\text { Median } \\
(\text { Q1, Q3) }\end{array}$ & $\begin{array}{l}4.00(1.00, \\
7.00)\end{array}$ & $\begin{array}{l}3.00(2.00, \\
6.00)\end{array}$ & $\begin{array}{c}3.00(1.00, \\
6.00)\end{array}$ & $\begin{array}{c}3.00(1.00, \\
5.00)\end{array}$ & $\begin{array}{l}2.00(1.00, \\
5.00)\end{array}$ & $\begin{array}{c}2.00(0.00, \\
4.00)\end{array}$ & $\begin{array}{l}2.00(0.00, \\
4.00)\end{array}$ & $\begin{array}{l}2.00(1.00, \\
5.00)\end{array}$ & \\
\hline Min-Max & $0.00-10.00$ & $0.00-10.00$ & $0.00-10.00$ & $0.00-10.00$ & $0.00-10.00$ & $0.00-10.00$ & $0.00-10.00$ & $0.00-10.00$ & \\
\hline \multicolumn{9}{|l|}{ Alcohol } & 0.051 \\
\hline $\begin{array}{l}\text { Non- } \\
\text { drinker }\end{array}$ & $149(54.2 \%)$ & $193(54.8 \%)$ & $225(48.3 \%)$ & $289(51.6 \%)$ & $326(46.3 \%)$ & $390(46.7 \%)$ & $519(47.3 \%)$ & $\begin{array}{l}2091 \\
\quad(48.8 \%)\end{array}$ & \\
\hline Past drinker & $21(7.6 \%)$ & $29(8.2 \%)$ & $27(5.8 \%)$ & $39(7.0 \%)$ & $56(8.0 \%)$ & $61(7.3 \%)$ & $69(6.3 \%)$ & $302(7.0 \%)$ & \\
\hline $\begin{array}{l}\text { Current } \\
\text { drinker }\end{array}$ & $105(38.2 \%)$ & $130(36.9 \%)$ & $214(45.9 \%)$ & $232(41.4 \%)$ & $322(45.7 \%)$ & $384(46.0 \%)$ & $509(46.4 \%)$ & $\begin{array}{l}1896 \\
(44.2 \%)\end{array}$ & \\
\hline \multicolumn{9}{|l|}{ Smoking } & 0.042 \\
\hline $\begin{array}{l}\text { Non- } \\
\text { smoker }\end{array}$ & $183(66.5 \%)$ & $259(73.6 \%)$ & $328(70.4 \%)$ & $385(68.8 \%)$ & $466(66.2 \%)$ & $548(65.6 \%)$ & $730(66.5 \%)$ & $\begin{array}{l}2899 \\
(67.6 \%)\end{array}$ & \\
\hline Past smoker & $28(10.2 \%)$ & $45(12.8 \%)$ & $45(9.7 \%)$ & $76(13.6 \%)$ & $89(12.6 \%)$ & $109(13.1 \%)$ & $127(11.6 \%)$ & $519(12.1 \%)$ & \\
\hline $\begin{array}{l}\text { Current } \\
\text { smoker }\end{array}$ & $64(23.3 \%)$ & $48(13.6 \%)$ & $93(20.0 \%)$ & $99(17.7 \%)$ & $149(21.2 \%)$ & $178(21.3 \%)$ & $240(21.9 \%)$ & $871(20.3 \%)$ & \\
\hline \multicolumn{9}{|l|}{ BMI } & 0.011 \\
\hline Mean (SD) & $23.17(2.89)$ & $23.09(2.59)$ & $23.49(2.60)$ & $23.13(2.57)$ & $23.43(2.52)$ & $23.50(2.43)$ & $23.25(2.37)$ & $23.32(2.51)$ & \\
\hline $\begin{array}{l}\text { Median } \\
\quad(\mathrm{Q} 1, \mathrm{Q} 3)\end{array}$ & $\begin{array}{l}22.89(21.45 \\
25.00)\end{array}$ & $\begin{array}{l}22.86(21.46 \\
24.63)\end{array}$ & $\begin{array}{l}23.48(21.78 \\
24.97)\end{array}$ & $\begin{array}{l}23.17(21.35 \\
24.61)\end{array}$ & $\begin{array}{l}23.31(21.64 \\
24.97)\end{array}$ & $\begin{array}{l}23.39 \text { (21.94, } \\
24.91)\end{array}$ & $\begin{array}{l}23.18(21.72 \\
24.54)\end{array}$ & $\begin{array}{l}23.23(21.64 \\
24.80)\end{array}$ & \\
\hline Min-Max & $15.40-36.11$ & $16.00-31.64$ & $17.26-33.98$ & $13.15-32.89$ & $16.82-31.63$ & $16.69-31.53$ & $16.33-34.48$ & $13.15-36.11$ & \\
\hline \multicolumn{9}{|c|}{ Arthritis or rheumatism } & $<0.001$ \\
\hline No & $208(75.6 \%)$ & $284(80.7 \%)$ & $352(75.5 \%)$ & $480(85.7 \%)$ & $584(83.0 \%)$ & $725(86.8 \%)$ & $995(90.7 \%)$ & $\begin{array}{l}3628 \\
\quad(84.6 \%)\end{array}$ & \\
\hline Yes & $67(24.4 \%)$ & $68(19.3 \%)$ & $114(24.5 \%)$ & $80(14.3 \%)$ & $120(17.0 \%)$ & $110(13.2 \%)$ & $102(9.3 \%)$ & $661(15.4 \%)$ & \\
\hline \multicolumn{9}{|l|}{ Mental illness } & 0.376 \\
\hline No & $272(98.9 \%)$ & $344(97.7 \%)$ & $462(99.1 \%)$ & $552(98.6 \%)$ & $695(98.7 \%)$ & $821(98.3 \%)$ & $\begin{array}{l}1088 \\
\quad(99.2 \%)\end{array}$ & $\begin{array}{l}4234 \\
\quad(98.7 \%)\end{array}$ & \\
\hline Yes & $3(1.1 \%)$ & $8(2.3 \%)$ & $4(0.9 \%)$ & $8(1.4 \%)$ & $9(1.3 \%)$ & $14(1.7 \%)$ & $9(0.8 \%)$ & $55(1.3 \%)$ & \\
\hline \multicolumn{9}{|l|}{ Liver diseases } & 0.135 \\
\hline No & $269(97.8 \%)$ & $346(98.3 \%)$ & $463(99.4 \%)$ & $547(97.7 \%)$ & $687(97.6 \%)$ & $818(98.0 \%)$ & $\begin{array}{l}1085 \\
(98.9 \%)\end{array}$ & $\begin{array}{l}4215 \\
\quad(98.3 \%)\end{array}$ & \\
\hline Yes & $6(2.2 \%)$ & $6(1.7 \%)$ & $3(0.6 \%)$ & $13(2.3 \%)$ & $17(2.4 \%)$ & $17(2.0 \%)$ & $12(1.1 \%)$ & $74(1.7 \%)$ & \\
\hline \multicolumn{9}{|l|}{ Lung diseases } & 0.15 \\
\hline No & $269(97.8 \%)$ & $342(97.2 \%)$ & $453(97.2 \%)$ & $548(97.9 \%)$ & $696(98.9 \%)$ & $818(98.0 \%)$ & $\begin{array}{l}1084 \\
(98.8 \%)\end{array}$ & $\begin{array}{l}4210 \\
\quad(98.2 \%)\end{array}$ & \\
\hline Yes & $6(2.2 \%)$ & $10(2.8 \%)$ & $13(2.8 \%)$ & $12(2.1 \%)$ & $8(1.1 \%)$ & $17(2.0 \%)$ & $13(1.2 \%)$ & $79(1.8 \%)$ & \\
\hline \multicolumn{9}{|l|}{ Cancer } & 0.487 \\
\hline No & $267(97.1 \%)$ & $338(96.0 \%)$ & $456(97.9 \%)$ & $540(96.4 \%)$ & $687(97.6 \%)$ & $816(97.7 \%)$ & $\begin{array}{l}1070 \\
(97.5 \%)\end{array}$ & $\begin{array}{l}4174 \\
(97.3 \%)\end{array}$ & \\
\hline Yes & $8(2.9 \%)$ & $14(4.0 \%)$ & $10(2.1 \%)$ & $20(3.6 \%)$ & $17(2.4 \%)$ & $19(2.3 \%)$ & $27(2.5 \%)$ & $115(2.7 \%)$ & \\
\hline \multicolumn{9}{|l|}{ Diabetes } & $<0.001$ \\
\hline No & $232(84.4 \%)$ & $297(84.4 \%)$ & $404(86.7 \%)$ & $503(89.8 \%)$ & $640(90.9 \%)$ & $750(89.8 \%)$ & $\begin{array}{l}1016 \\
(92.6 \%)\end{array}$ & $\begin{array}{l}3842 \\
(89.6 \%)\end{array}$ & \\
\hline Yes & $43(15.6 \%)$ & $55(15.6 \%)$ & $62(13.3 \%)$ & $57(10.2 \%)$ & $64(9.1 \%)$ & $85(10.2 \%)$ & $81(7.4 \%)$ & $447(10.4 \%)$ & \\
\hline \multicolumn{9}{|c|}{ High blood pressure } & $<0.001$ \\
\hline No & $185(67.3 \%)$ & $257(73.0 \%)$ & $316(67.8 \%)$ & $430(76.8 \%)$ & $516(73.3 \%)$ & $622(74.5 \%)$ & $895(81.6 \%)$ & $\begin{array}{l}3221 \\
\quad(75.1 \%)\end{array}$ & \\
\hline Yes & $90(32.7 \%)$ & $95(27.0 \%)$ & $150(32.2 \%)$ & $130(23.2 \%)$ & $188(26.7 \%)$ & $213(25.5 \%)$ & $202(18.4 \%)$ & $\begin{array}{l}1068 \\
(24.9 \%)\end{array}$ & \\
\hline
\end{tabular}

Total number of chronic diseases

$<0.001$ 
Table 1 (continued)

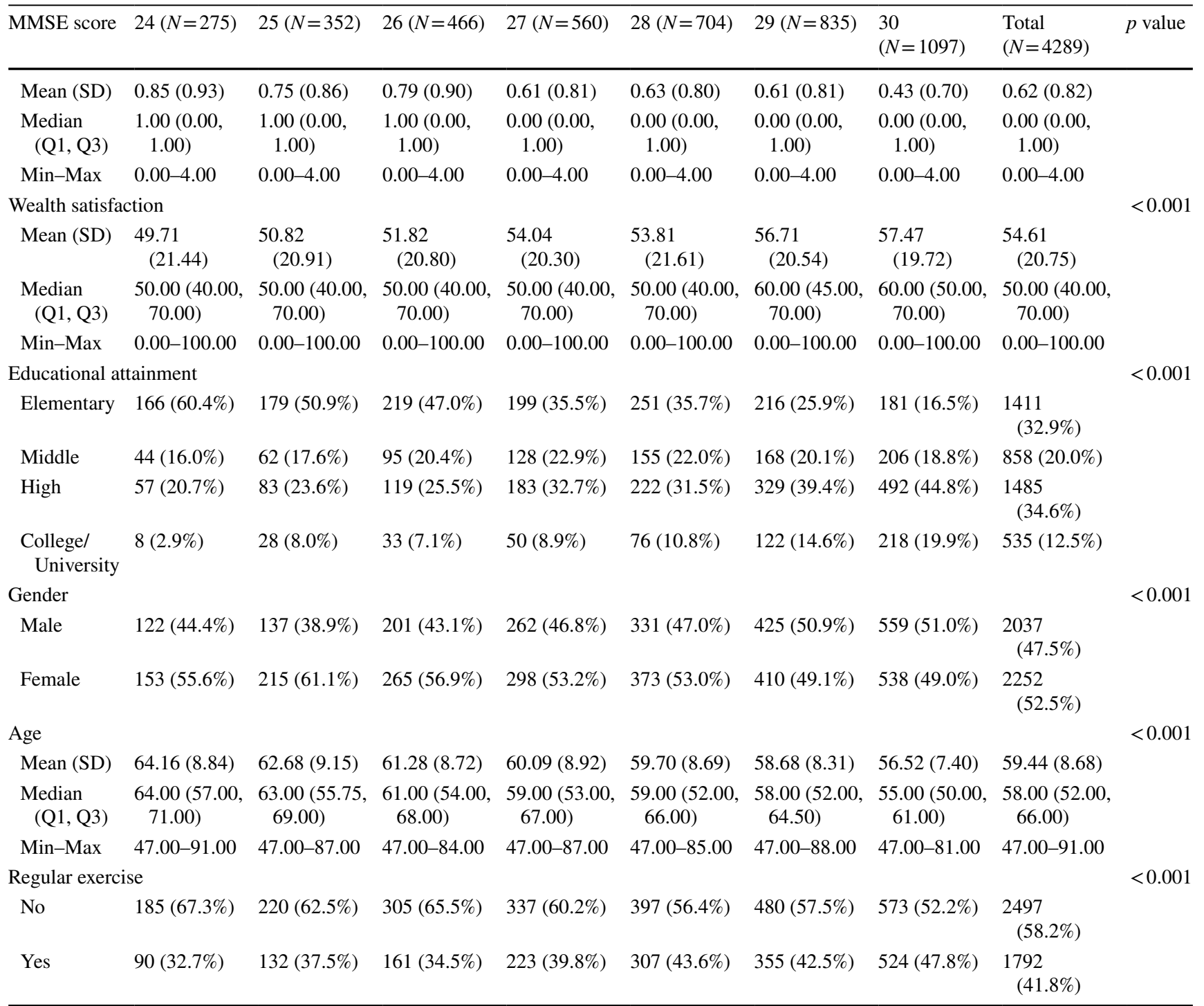

10 variables out of 17 showed statistical significance when tested for correlation with MMSE score. These variables were $C E S D$-10, presence of joint conditions; diabetes; HBP conditions, total number of chronic diseases (sumchron), wealth satisfaction, highest education level, gender, age, and regular exercise behaviors

\section{Results}

A descriptive analysis of the participants at the beginning of this study in relation to each MMSE score (1-30) can be found in Table 1.

Figures 2 and 3 display the univariate Cox proportional hazard regression models for six selected modifiable factors. The drinking and smoking status of the respondents (which were not statistically significant in Chi-square test) did not have a significant protective effect on the event of cognitive decline over time (for model 1 or 2). However, high scores on CESD-10, higher total number of chronic diseases ( sumchron), presence of $H B P$, and respondents without regular exercise showed risks of developing both dementia and MCI.
Table 2 represents adjusted hazard ratios (aHR) of Cox mixed-effects proportional hazard regression of dementia (model 1) and MCI (model 2). Model 1 uses maximumlikelihood ratio tests based on the integrated and penalized views of the model, along with penalized values. The hazard ratios presented are the results after controlling individual effects as well as other co-variates. A variance of 1.81 (with standard deviation of 1.34) was observed in model 1, while a variance of 0.65 (with standard deviation of 0.80 ) was observed in model 2.

In Model 1 , the statistically significant $(P<0.05)$ adverse factors associated with the development of dementia were age (aHR 1.07, 1.05-1.09), CESD-10 (each unit increase accounts for aHR 1.17, 1.12-1.23), diagnosis 

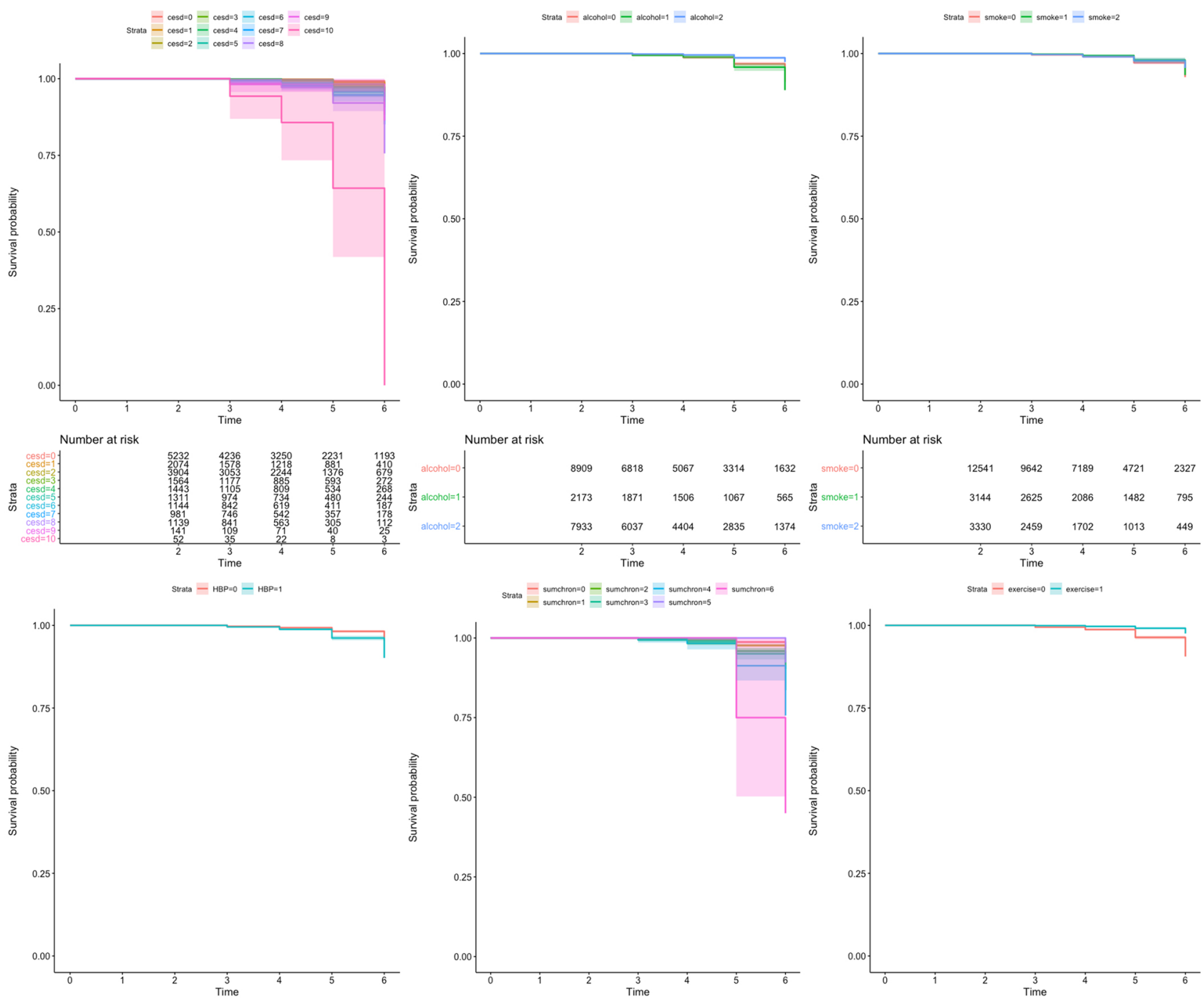

Strata $=$ exercise $=0$ exercisen 1
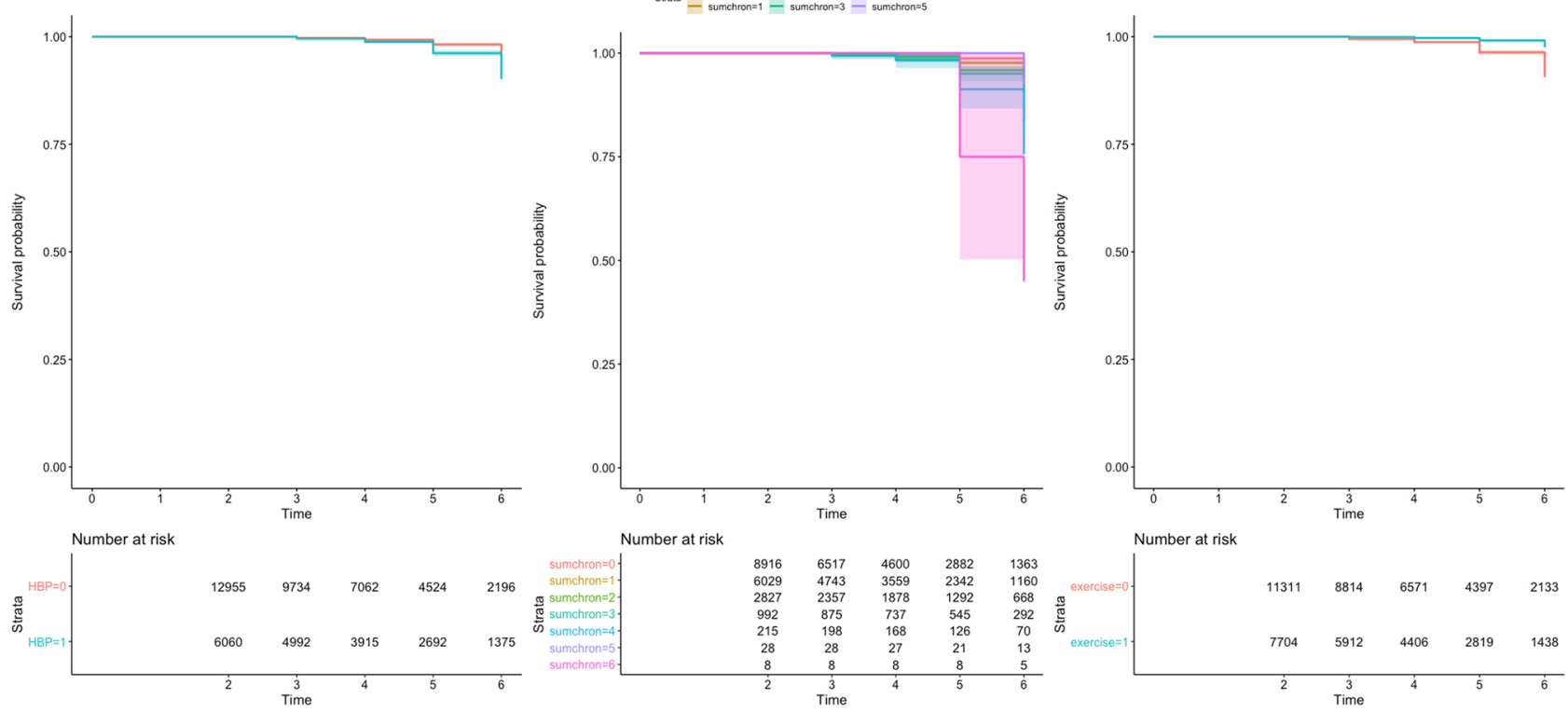

Fig. 2 Model 1: the univariate Cox proportional hazard regression models for six modifiable factors using MMSE score cut-off for dementia $(\leq 17)$ as a hazard outcome, five cumulative waves $(2-6)$

with cerebrovascular disease (aHR 3.73, 1.81-7.66), living with diabetes (aHR 2.30, 1.22-4.35), and living with HBP (aHR 2.05, 1.09-3.87). In contrast, the statistically significant protective factors against developing dementia were current alcohol consumption (aHR 0.67, 0.46-0.99), increase in wealth satisfaction (each unit increase in satisfaction accounts for aHR 0.98, 0.98-0.99), higher educational attainment (aHR 0.36, 0.26-0.56), and regular exercise (aHR 0.37, 0.26-0.51).

In Model 2, the statistically significant adverse factors that affected the event of decline in cognitive ability to MCI were age (aHR 1.02, 1.01-1.03) and CESD-10 (each unit increase accounts for aHR 1.17, 1.14-1.19). In contrast, the statistically significant protective factors against developing MCI were BMI (every unit increase in $\mathrm{kg} / \mathrm{m}^{2}$ accounts for aHR 0.96, 0.94-0.98), increase in wealth satisfaction (each unit increase in satisfaction accounts for aHR 0.99, 0.99-0.99), higher educational attainment (aHR 0.33, 0.26-0.43), and regular exercise (aHR 0.83, 0.74-0.92). 

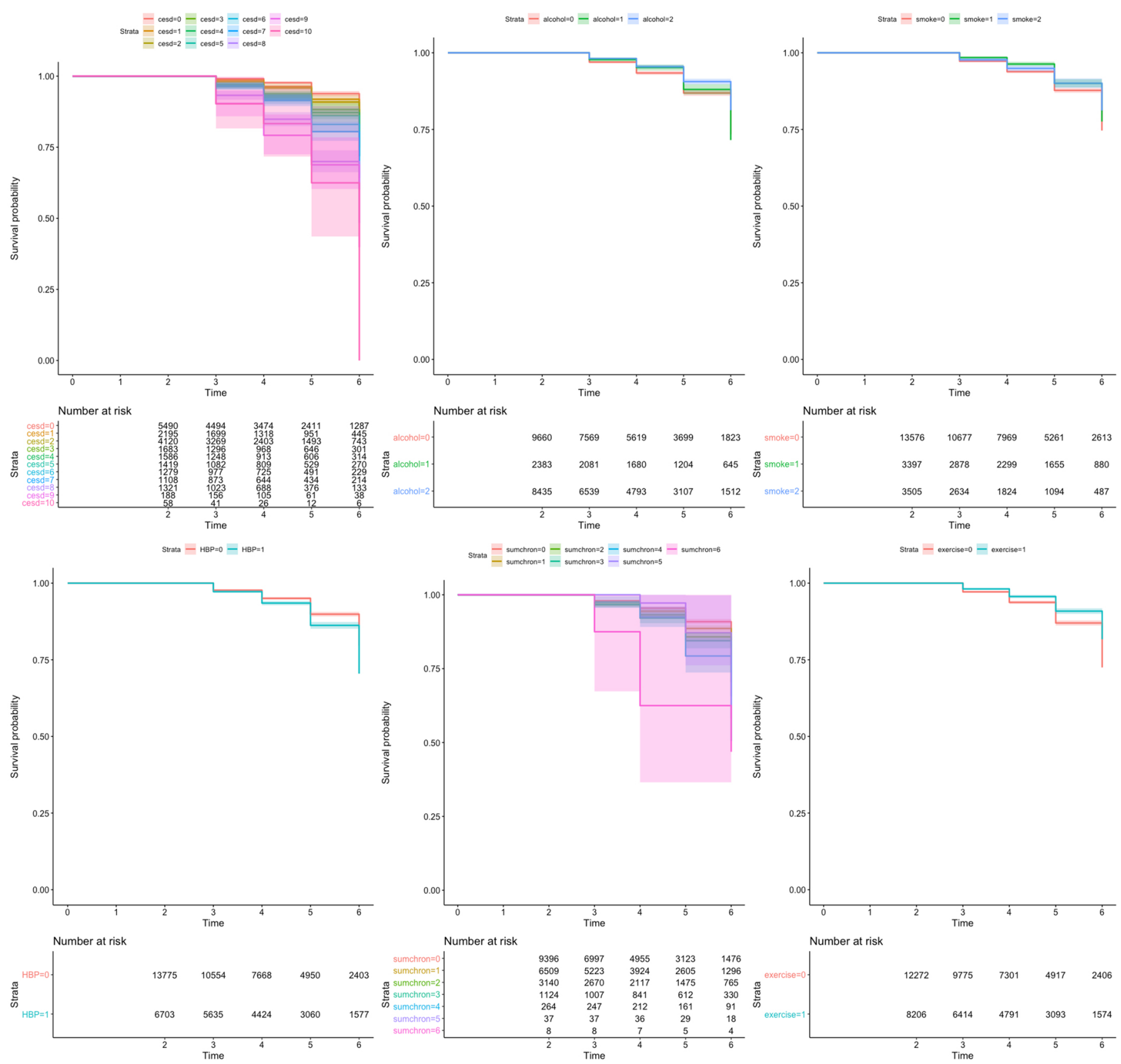

Fig. 3 Model 2: the univariate Cox proportional hazard regression models for six modifiable factors using MMSE score cut-off for MCI $(18 \leq \mathrm{x} \leq 23)$ as a hazard outcome, five cumulative waves $(2-6)$

\section{Discussion}

Using data from this large cohort representative of the Korea population, aged 45 or older who were free from pre-existing cerebrovascular disease and CHD, we observed several factors that contribute significantly in developing dementia (model 1). When adjusting for age, lifestyle factors, socioeconomic determinates, and existing illnesses, being newly diagnosed with cerebrovascular disease increased the risk of developing dementia 3.73 times. While not statistically significant, being newly diagnosed with CHD increases the risk
1.3 times compared to those who do not. Almost all existing chronic diseases increased the risk of dementia (after adjustment), with existing diabetes condition showing the greatest statistically significant risk for developing dementia (2.3 times more than those who do not have diabetes). Existing HBP condition also showed great risks in developing dementia; those who have HBP were 2.05 times more likely to develop dementia. In addition, arthritis and rheumatism also increased the risks of developing dementia, which contrasts with the lifestyle factor of regular exercise-which may suggest reverse causality due to limited movement and joint pain leading to reduced ability to exercise routinely. Similar 
Table 2 Adjusted hazard ratios of Cox mixed-effects proportional hazard regression of dementia (model 1) and MCI (model 2)

\begin{tabular}{|c|c|c|}
\hline & Model 1: hazard ratio (adjusted) & Model 2: hazard ratio (adjusted) \\
\hline \multicolumn{3}{|l|}{ Gender (ref: male) } \\
\hline Female & $1.26(0.82-1.93)$ & $1.10(0.92-1.31)$ \\
\hline Age & $1.07 * * *(1.05-1.09)$ & $1.02 * * *(1.01-1.03)$ \\
\hline BMI $\left(\mathrm{kg} / \mathrm{m}^{2}\right)$ & $0.96(0.92-1.01)$ & $0.96 * * *(0.94-0.98)$ \\
\hline CESD-10 (Min:0, Max:10) & $1.17 * * *(1.12-1.23)$ & $1.17 * * *(1.14-1.19)$ \\
\hline \multicolumn{3}{|l|}{ Alcohol (ref: non-drinker) } \\
\hline Past drinker & $1.26(0.87-1.84)$ & $0.96(0.81-1.14)$ \\
\hline Current drinker & $0.67 * *(0.46-0.99)$ & $0.94(0.81-1.08)$ \\
\hline \multicolumn{3}{|l|}{ Smoke (ref: non-smoker) } \\
\hline Past smoker & $1.08(0.69-1.70)$ & $0.94(0.78-1.14)$ \\
\hline Current smoker & $1.42(0.86-2.34)$ & $0.96(0.78-1.18)$ \\
\hline \multicolumn{3}{|c|}{ Newly diagnosed with cardiovascular diseases (ref: never diagnosed) } \\
\hline Coronary heart disease & $1.30(0.56-3.02)$ & $1.00(0.71-1.41)$ \\
\hline Cerebrovascular disease & $3.73 * * *(1.81-7.66)$ & $1.00(0.68-1.47)$ \\
\hline \multicolumn{3}{|l|}{ Living with chronic illness (ref: absent) } \\
\hline Arthritis or rheumatism & $1.81 *(0.95-3.43)$ & $1.06(0.81-1.38)$ \\
\hline Mental illness & $1.42(0.62-3.29)$ & $1.03(0.71-1.48)$ \\
\hline Liver diseases & $0.73(0.22-2.43)$ & $1.06(0.71-1.59)$ \\
\hline Lung diseases & $1.53(0.61-3.85)$ & $0.95(0.64-1.39)$ \\
\hline Cancer & $1.81(0.84-3.90)$ & $0.79(0.57-1.10)$ \\
\hline Diabetes & $2.30 * *(1.22-4.35)$ & $0.94(0.72-1.23)$ \\
\hline High blood pressure & $2.05 * *(1.09-3.87)$ & $1.13(0.88-1.47)$ \\
\hline Wealth satisfaction (Min:0, Max:10) & $0.98 * * *(0.98-0.99)$ & $0.99 * * *(0.99-0.99)$ \\
\hline \multicolumn{3}{|c|}{ Educational attainment (ref: Elementary school) } \\
\hline Middle school & $0.46 * * *(0.31-0.69)$ & $0.64 * * *(0.55-0.74)$ \\
\hline High school & $0.38 * * *(0.26-0.56)$ & $0.42 * * *(0.36-0.48)$ \\
\hline College/university & $0.40 * * *(0.21-0.75)$ & $0.33 * * *(0.26-0.43)$ \\
\hline Regular exercise (ref: absent) & $0.37 * * *(0.26-0.51)$ & $0.83 * * *(0.74-0.92)$ \\
\hline
\end{tabular}

$* * * p<0.01, * * p<0.05, * p<0.1$

trends were shown in MCI (model 2), with some exceptions of liver diseases, lung diseases, cancer, and diabetes. Therefore, it was noted that while liver diseases may accelerate cognitive decline before the MCI (rather than after), lung diseases, cancer, and diabetes do not contribute to cognitive decline until the patients reach their MCI stage. Being newly diagnosed with CHD or cerebrovascular disease also shows effects only after MCI stage is reached, but not before.

This is in agreement with White and colleagues who found through post-mortem examinations a strong association between dementia and death from cerebrovascular disease [64]. The association between HBP and increased risk of dementia and MCI in later life is similar to a recent nationwide cohort study in Korea, in which they found a linear association between increased blood pressure and all-type dementia [65]. The association of diabetes with a higher risk in all-cause dementia also agrees with the majority of the literature and other Korean countrywide population studies [35], while the difference in the results between two models may be due to variation in diabetes management [66]. For instances, patients who already faced MCI may have lack of capacity in managing their diabetes compared to those who have not. While diabetes may not advance cognitive decline before MCI, if a diabetes management plan is well followed, the worsening of diabetic conditions due to MCI can accelerate further cognitive decline and contribute to dementia.

Interestingly, while each unit increase in severity of depressive symptoms (CESD-10) increased the risks of developing both MCI and dementia, when we controlled the depressive symptoms, both alcohol status and smoking status showed protective effects to MCI. However, it is important to consider that drinking and smoking variables had a three-level classification in this study-whilst there can often be a great difference in the level of consumption of alcohol or number of cigarettes smoked per week. As well, heavy drinkers and smokers often have a significantly shorter life expectancy and can die before the late-life 
manifestation of MCI $[67,68]$. Moreover, the present study could demonstrate a pattern of lower dementia risk for past smokers when compared to current smokers which aligns with numerous studies $[69,70]$. The lack of a significant difference in this study could be attributed to variation in the period of smoking cessation. Another longitudinal study by Choi and colleagues found a significant decrease risk for dementia in long-term past smokers' Korean men, however, not for short-term quitters [33]. The assessment of the smoking cessation period is required in future studies.

In contrast, in terms of risks of developing dementia, past drinkers showed higher risks than current drinkers. While the lack of association between current drinking and dementia could be due to differences in the volume of alcohol consumption, low to moderate levels of alcohol consumption has been suggested to have no effect [71] or even decreased risk for dementia and cognitive decline [72]. The potential neuroprotective effects could be attributed reducing the effects other of CVD risk factors and a healthy lifestyle pattern [73], and polyphenols in Korean rice wine [74, 75]. However, the negative effects of chronic high alcohol consumption may supersede any potential positive effects [76]. An extensive umbrella review found an association of excess alcohol consumption and dementia [77]. Moreover, former drinkers may have stopped their alcohol consumption due to the health consequences [78], and this could overestimate the benefits of continued alcohol use and increased risk when compared to those who never drink. oftentimes, individuals may cease drinking because of sickness that may be unobserved in the study, leaving former drinkers with higher risk. In addition, current drinkers with alcohol use disorders (AUD) are often underrepresented and have substantially shortened lifespan compared to former AUD individuals [79, 80]. A previous diagnosis of AUD has a greater risk for all-cause dementia than all other modifiable risk factors [77]. Therefore, the adverse effects of drinking heavily before the age of 45 may be reflected in their cognitive decline over time [81]. In addition, these results can reflect an institution's policy of no drinking or no smoking for the participants who are residing in institutions (i.e., care homes).

High BMI was also a protective factor in developing dementia and MCI, in agreement with previous research [82-85]. This mirrors the descriptive analysis and the literature which showed a low prevalence of obese and overweight Koreans born before 1970 [86]. The protective effect of a high BMI may be attributed reverse causality, since prodromal dementia and MCI are associated with weight loss [87]. In contrast, it is also important to note that higher BMI can reduce life expectancy and individuals will pass away before the development of dementia [88]. Nevertheless, obesity's association with cognitive impairment and dementia has been contradictory; while mid-life obesity has been identified as a risk factor [58], it was observed to be protective in later life [59]. In one Korean-based population study, late-life obesity was associated with dementia [60], while another study found no association between baseline BMI and dementia, except in underweight males [61]. The fundamental relationship between BMI and dementia is not conclusive in Korean populations. On the other hand, key limitations in using the BMI measurement for obesity is that it does not distinguish between lean muscle and fat, and underestimates adiposity in the elderly [89]. Further studies should consider supplementary measurements (skinfold thickness and waist circumstance) to better determine obesity status. Finally, both high educational and occupational attainment have been suggested to contribute to a "cognitive reserve" that can slow down cognitive decline related to $\mathrm{AD}$ and delay onset [90]. This effect was also observed in our study, having higher education, greater wealth satisfaction, and regular exercise behaviors showed statistically significant protective results in developing both dementia and MCI, most likely due to practicing healthy lifestyle as a result of having higher socio-economic positions and resources being available for them to practice such protective factors.

\section{Limitations}

In this study, a list-wise deletion was used to handle the missing values and dropout before wave 6 . Since the missing values are likely to be Missing-Not-At-Random (MNAR) due to the participants' desire not to give out a sensitive information about themselves, it can underestimate the association between the risk factors to dementia and MCI. Also, the using list-wise deletion to treat missing data may have deleted the important data that may greatly influence the outcome variables (thus, creating a selection bias and attrition bias). However, out of whole dataset, only nine variables had missingness at wave 2 and each variable showed less than $7 \%$ of missingness. In addition, retention rate from wave 2 to 6 was $76 \%$, which is above critical value (70\%). Another limitation is that despite both participants' surveys and health professionals' opinion on medical/biological conditions used in KLoSA, the participants may suffer from recall bias or misinterpretation during the interview, which could have led to random misclassification and bias toward the null value.

A fundamental limitation is that this study only covers ten years, while often extended preclinical duration of dementia can take up to 20 years before diagnosis. It may be an insufficient period for symptoms to manifest from a mid-life population. Further continuation of this cohort study would overcome this limitation and ameliorate possible reverse causality. The MMSE test is also insufficient to diagnosis or exclude dementia entirely, and to differentiate between MCI and dementia. A study by Hensel and colleagues showed that the reliable change 
between wave 1-5 of the longitudinal study on the MMSE score is -3 to +3 , [50]. This may lead to low sensitivity in detecting MCI and can be also due to the test's lack of complexity as well as the absence of executive function items [51]. In addition, MMSE does not consider patient's mood on the day when they take the test and it is possible that patients may be disrupted due to their current mental state, for example, depression or anxiety [52]. However, this has been controlled to the highest level as possible by controlling different co-variates affecting the MMSE scores including, depression scale and educational attainment. However, multiple MMSE tests over time can be used to predict the conversion of MCI to dementia [49], which was performed during the 10 years of data collection of KLoSA. While the subtype of the dementia is not identified in this study, limiting the association of the factors with a specific type, the factors associated with allcause dementia are likely to be similar to AD with it being the majority of Korean dementia cases [32]. Moreover, the MMSE test provided an easy and quick administration, and is extensively used to distinguish between dementia and normal cognition.

\section{Conclusion}

This study found that new diagnosis with CVDs (cerebrovascular disease and CHD) greatly increased the risks of developing dementia in a Korean population, while being diagnosed with CVDs before MCI had no effect on cognitive decline. This suggests that preventing patients with MCI from developing CVDs is crucial in delaying their cognitive decline rapidly advancing to dementia. Also, existing HBP and/or arthritis or rheumatism conditions increased the risks of developing cognitive decline leading to both MCI and dementia. In lieu of the protective factor of MCI and dementia, implementing regular exercise routine well before mid-life and cognitive decline is significant, with adjustments made for those suffering from health conditions, so they can continue exercising despite their morbidity. Finally, further attention in diabetes care and management is needed for patients who already show decline in cognitive ability, as it is likely that their MCI affect their ability to manage their existing chronic conditions and may adversely affect their cognitive ability further.

Funding This research received no specific grant from any funding agency in the public, commercial, or not-for-profit sectors.
Data availability The datasets generated during and/or analyzed during the current study are available from the corresponding author on reasonable request.

Code availability The codes generated during and/or analyzed during the current study are available from the corresponding author on reasonable request.

\section{Compliance with ethical standards}

Conflicts of interest The authors have no conflicts of interest to declare that are relevant to the content of this article.

Ethical approval The survey of Korean Longitudinal Study of Aging (KLoSA) was approved by Institutional Review Board of the Korea Centers for Disease Control and Prevention. In addition, as KloSA database is released to the public for scientific use, ethical approval was not needed for this study.

Statement of human and animal rights The study was designed in conformity with the Declaration of Helsinki.

Informed consent All participants gave their informed written consent.

Open Access This article is licensed under a Creative Commons Attribution 4.0 International License, which permits use, sharing, adaptation, distribution and reproduction in any medium or format, as long as you give appropriate credit to the original author(s) and the source, provide a link to the Creative Commons licence, and indicate if changes were made. The images or other third party material in this article are included in the article's Creative Commons licence, unless indicated otherwise in a credit line to the material. If material is not included in the article's Creative Commons licence and your intended use is not permitted by statutory regulation or exceeds the permitted use, you will need to obtain permission directly from the copyright holder. To view a copy of this licence, visit http://creativecommons.org/licenses/by/4.0/.

\section{References}

1. WHO (2016) Child Mortal causes death WHO. WHO, Geneva

2. World Health Organization (2017) Cardiovascular diseases (CVDs). WHO, Geneva

3. Divo M, Cote C, de Torres JP et al (2012) Comorbidities and risk of mortality in patients with chronic obstructive pulmonary disease. Am J Respir Crit Care Med 186:155-161

4. World Heart Federation (2017) Risk factors. https://www.world -heart-federation.org/resources/risk-factors/. Accessed 3 Jun 2020

5. de Lange AH, Taris TW, Kompier MAJ et al (2005) Different mechanisms to explain the reversed effects of mental health on work characteristics. Scand J Work Environ Health. https://doi. org/10.5271/sjweh.843

6. Kagan AR, Levi L (1974) Health and environment-psychosocial stimuli: a review. Soc Sci Med 8:225-241

7. De Jonge J, Dormann C, Janssen PPM et al (2001) Testing reciprocal relationships between job characteristics and psychological well-being: A cross-lagged structural equation model. J Occup Organ Psychol 74:29-46

8. Marmot M, Allen J, Bell R et al (2012) WHO European review of social determinants of health and the health divide. Lancet 380:1011-1029 
9. Cockerham WC, Hamby BW, Oates GR (2017) The social determinants of chronic disease. Am J Prev Med. https://doi. org/10.1016/j.amepre.2016.09.010

10. Irwin A, Valentine N, Brown $C$ et al (2006) The commission on social determinants of health: tackling the social roots of health inequities. PLoS Med 3:e106

11. World Health Organization (2019) Statement on the meeting of the International Health Regulations (2005) Emergency Committee regarding the outbreak of novel coronavirus 2019 (n-CoV). World Health Organization, Geneva

12. Prince M, Ali G-C, Guerchet M et al (2016) Recent global trends in the prevalence and incidence of dementia, and survival with dementia. Alzheimers Res Ther 8:23

13. Crous-Bou M, Minguillón C, Gramunt N et al (2017) Alzheimer's disease prevention: from risk factors to early intervention. Alzheimers Res Ther 9:71

14. Hugo J, Ganguli M (2014) Dementia and cognitive impairment: epidemiology, diagnosis, and treatment. Clin Geriatr Med 30:421-442

15. Jellinger KA, Attems J (2010) Prevalence of dementia disorders in the oldest-old: An autopsy study. Acta Neuropathol 119:421-433. https://doi.org/10.1007/s00401-010-0654-5

16. Petersen RC, Roberts RO, Knopman DS et al (2009) Mild cognitive impairment: ten years later. Arch Neurol 66:1447-1455

17. Okonkwo OC, Griffith HR, Copeland JN et al (2008) Medical decision-making capacity in mild cognitive impairment: a 3-year longitudinal study. Neurology 71:1474-1480

18. Knopman DS, Petersen RC (2014) Mild cognitive impairment and mild dementia: a clinical perspective. Mayo Clin Proc 89:14521459. https://doi.org/10.1016/j.mayocp.2014.06.019

19. Langa KM, Levine DA (2014) The diagnosis and management of mild cognitive impairment: a clinical review. JAMA 312:2551-2561

20. Burns JM, Jeffrey M, Morris JC (2008) Mild cognitive impairment and early Alzheimer's disease : detections and diagnosis. John Wiley \& Sons, Hoboken

21. Kuller LH, Lopez OL, Newman A et al (2003) Risk factors for dementia in the cardiovascular health cognition study. Neuroepidemiology 22:13-22. https://doi.org/10.1159/000067109

22. Whitmer RA, Sidney S, Selby J et al (2005) Midlife cardiovascular risk factors and risk of dementia in late life. Neurology 64:277281. https://doi.org/10.1212/01.WNL.0000149519.47454.F2

23. Alonso A, Jacobs DR, Menotti A et al (2009) Cardiovascular risk factors and dementia mortality: 40 years of follow-up in the Seven Countries Study. J Neurol Sci 280:79-83. https://doi. org/10.1016/j.jns.2009.02.004

24. Rakesh G, Szabo ST, Alexopoulos GS et al (2017) Strategies for dementia prevention: latest evidence and implications. Ther Adv Chronic Dis 8:121-136

25. Leritz EC, McGlinchey RE, Kellison I et al (2011) Cardiovascular disease risk factors and cognition in the elderly. Curr Cardiovasc Risk Rep 5:407

26. Biessels GJ, Reijmer YD (2014) Brain changes underlying cognitive dysfunction in diabetes: what can we learn from MRI? Diabetes 63:2244-2252

27. Kyu HH, Pinho C, Wagner JA et al (2016) Global and national burden of diseases and injuries among children and adolescents between 1990 and 2013. JAMA Pediatr 170:267. https://doi. org/10.1001/jamapediatrics.2015.4276

28. Song R, Xu H, Dintica CS et al (2020) Associations between cardiovascular risk, structural brain changes, and cognitive decline. J Am Coll Cardiol 75:2525-2534

29. Livingston G, Huntley J, Sommerlad A et al (2020) Dementia prevention, intervention, and care: 2020 report of the Lancet Commission. Lancet 396:413-446
30. Ritchie K, Carriere I, Su L et al (2017) The midlife cognitive profiles of adults at high risk of late-onset Alzheimer's disease: The PREVENT study. Alzheimer's Dement 13:1089-1097

31. Irwin K, Sexton C, Daniel $T$ et al (2018) Healthy aging and dementia: two roads diverging in midlife? Front Aging Neurosci 10:275

32. Kim YJ, Han JW, So YS et al (2014) Prevalence and trends of dementia in Korea: a systematic review and meta-analysis. J Korean Med Sci 29:903-912

33. Choi D, Choi S, Park SM (2018) Effect of smoking cessation on the risk of dementia: a longitudinal study. Ann Clin Transl Neurol 5:1192-1199

34. Lee D, Kim B-J, Han JW et al (2020) Low diastolic blood pressure and cognitive decline in korean elderly people: the korean longitudinal study on cognitive aging and dementia. Psychiatry Investig 17:21

35. Yu JH, Han K, Park S et al (2020) Incidence and risk factors for dementia in type 2 diabetes mellitus: a nationwide populationbased study in Korea. Diabetes Metab J 44:113-124. https://doi. org/10.4093/dmj.2018.0216

36. Jung S-J, Park S-H, Choi E-K et al (2014) Beneficial effects of Korean traditional diets in hypertensive and type 2 diabetic patients. J Med Food 17:161-171

37. Kim Y, Han B-G, Group K (2017) Cohort profile: the Korean genome and epidemiology study (KoGES) consortium. Int J Epidemiol 46:e20-e20

38. Hoshide S, Wang J-G, Park S et al (2016) Treatment considerations of clinical physician on hypertension management in Asia. Curr Hypertens Rev 12:164-168

39. Rizzi L, Rosset I, Roriz-Cruz M (2014) Global epidemiology of dementia: Alzheimer's and vascular types. Biomed Res Int. https ://doi.org/10.1155/2014/908915

40. Lee HJ, Jang J, Choi D-W et al (2020) Association between change in lifestyle and cognitive functions among elderly Koreans: findings from the Korean longitudinal study of aging (2006-2016). BMC Geriatr 20:1-12

41. Moon OR, Kim NS, Jang SM et al (2002) The relationship between body mass index and the prevalence of obesity-related diseases based on the 1995 National Health Interview Survey in Korea. Obes Rev 3:191-196

42. Shin SJ, Lim CY, Rhee M-Y et al (2011) Characteristics of sodium sensitivity in Korean populations. J Korean Med Sci 26:1061-1067

43. Rhee M-Y, Yang SJ, Oh SW et al (2011) Novel genetic variations associated with salt sensitivity in the Korean population. Hypertens Res 34:606-611

44. Korea Employment Information Service (2018) KLoSA (Korean Longitudinal Study of Aging). https://survey.keis.or.kr/eng/klosa /codebook/List.jsp. Accessed 4 Jun 2020

45. Kang H (2013) The prevention and handling of the missing data. Korean J Anesthesiol 64:402

46. Folstein MF, Folstein SE, Mchugh PR (1975) "Mini-mental state": A practical method for grading the cognitive state of patients for the clinician. J Psychiatr Res 12:189-198

47. Tombaugh TN, McIntyre NJ (1992) The mini-mental state examination: a comprehensive review. J Am Geriatr Soc 40:922-935. https://doi.org/10.1111/j.1532-5415.1992.tb01992.x

48. Ostrosky-Solís F, López-Arango G, Ardila A (2000) Sensitivity and specificity of the mini-mental state examination in a spanishspeaking population. Appl Neuropsychol 7:25-31. https://doi. org/10.1207/S15324826AN0701_4

49. Arevalo-Rodriguez I, Smailagic N, Roqué i Figuls M et al (2015) Mini-Mental State Examination (MMSE) for the detection of Alzheimer's disease and other dementias in people with mild cognitive impairment (MCI). Cochrane Database Syst Rev. https://doi. org/10.1002/14651858.CD010783.pub2 
50. Hensel A, Angermeyer MC, Riedel-Heller SG (2007) Measuring cognitive change in older adults: Reliable change indices for the Mini-Mental State Examination. J Neurol Neurosurg Psychiatry 78:1298-1303. https://doi.org/10.1136/jnnp.2006.109074

51. Trzepacz PT, Hochstetler H, Wang S et al (2015) Relationship between the montreal cognitive assessment and mini-mental state examination for assessment of mild cognitive impairment in older adults. BMC Geriatr 15:107. https://doi.org/10.1186/s1287 7-015-0103-3

52. Buckingham DN, Mackor KM, Miller RM et al (2013) Comparing the cognitive screening tools: MMSE and SLUMS. Pure Insights 2(1):3

53. Swan GE, Lessov-Schlaggar CN (2007) The effects of tobacco smoke and nicotine on cognition and the brain. Neuropsychol Rev $17: 259-273$

54. Wium-Andersen IK, Rungby J, Jørgensen MB et al (2020) Risk of dementia and cognitive dysfunction in individuals with diabetes or elevated blood glucose. Epidemiol Psychiatr Sci. https://doi. org/10.1017/S2045796019000374

55. Park K, Lim S, Park Y et al (2018) Cardiovascular disease risk factors and obesity levels in Korean adults: results from the Korea National Health and Nutrition Examination Survey, 2007-2015. Osong public Heal Res Perspect 9:150

56. Oh SW (2011) Obesity and metabolic syndrome in Korea. Diabetes Metab J 35:561-566

57. Uranga RM, Keller JN (2019) The complex interactions between obesity, metabolism and the brain. Front Neurosci 13:513

58. Tolppanen A-M, Ngandu T, Kåreholt I et al (2014) Midlife and late-life body mass index and late-life dementia: results from a prospective population-based cohort. J Alzheimer's Dis 38:201-209

59. Emmerzaal TL, Kiliaan AJ, Gustafson DR (2015) 2003-2013: a decade of body mass index, Alzheimer's disease, and dementia. J Alzheimer's Dis 43:739-755

60. Cho GJ, Hwang SY, Lee K et al (2019) Association between waist circumference and dementia in older persons: a nationwide population-based study. Obesity 27:1883-1891

61. Park S, Jeon S-M, Jung S-Y et al (2019) Effect of late-life weight change on dementia incidence: a 10-year cohort study using claim data in Korea. BMJ Open 9:e021739

62. Zhang R, Shen L, Miles T et al (2020) Association of low to moderate alcohol drinking with cognitive functions from middle to older age among US Adults. JAMA Netw open 3:e207922-e207922

63. Handing EP, Andel R, Kadlecova P et al (2015) Midlife alcohol consumption and risk of dementia over 43 years of follow-up: A population-based study from the Swedish twin registry. J Gerontol Ser A Biomed Sci Med Sci 70:1248-1254

64. White LON, Petrovitch H, Hardman J et al (2002) Cerebrovascular pathology and dementia in autopsied Honolulu-Asia Aging Study participants. Ann N Y Acad Sci 977:9-23

65. Yoo JE, Shin DW, Han K et al (2020) Blood pressure variability and the risk of dementia: a nationwide cohort study. Hypertension 75:982-990

66. Roberts RO, Geda YE, Knopman DS et al (2008) Association of duration and severity of diabetes mellitus with mild cognitive impairment. Arch Neurol 65:1066-1073

67. Westman J, Wahlbeck K, Laursen TM et al (2015) Mortality and life expectancy of people with alcohol use disorder in Denmark, Finland and Sweden. Acta Psychiatr Scand 131:297-306

68. Bernhard D, Moser C, Backovic A, Wick G (2007) Cigarette smoke-an aging accelerator? Exp Gerontol 42:160-165

69. Sonoda N, Morimoto A, Ugi S et al (2016) Smoking status is associated with mild cognitive impairment assessed with the minimental state examination in Japanese diabetic patients. Diabetol Int 7:361-367. https://doi.org/10.1007/s13340-016-0256-0
70. Lu Y, Sugawara Y, Zhang S et al (2020) Smoking cessation and incident dementia in elderly Japanese: the Ohsaki Cohort 2006 Study. Eur J Epidemiol 35:851-860. https://doi.org/10.1007/s1065 4-020-00612-9

71. Woods AJ, Porges EC, Bryant VE et al (2016) Current heavy alcohol consumption is associated with greater cognitive impairment in older adults. Alcohol Clin Exp Res 40:2435-2444

72. Wiegmann C, Mick I, Brandl EJ et al (2020) Alcohol and dementia-what is the link? A systematic review. Neuropsychiatr Dis Treat 16:87

73. Mostofsky E, Mukamal KJ, Giovannucci EL et al (2016) Key findings on alcohol consumption and a variety of health outcomes from the Nurses' Health Study. Am J Public Health 106:1586-1591

74. Lee J, Lee Y, Ha J et al (2018) Simultaneous determination of four bioactive compounds in Korean rice wine (makgeolli) by solvent extraction coupled with gas chromatography-mass spectrometry. Int J Food Prop 21:139-146

75. Lefèvre-Arbogast $\mathrm{S}$, Gaudout $\mathrm{D}$, Bensalem J et al (2018) Pattern of polyphenol intake and the long-term risk of dementia in older persons. Neurology 90:e1979-e1988

76. Koch M, Fitzpatrick AL, Rapp SR et al (2019) Alcohol consumption and risk of dementia and cognitive decline among older adults with or without mild cognitive impairment. JAMA Netw open 2:e1910319-e1910319

77. Rehm J, Hasan OSM, Black SE et al (2019) Alcohol use and dementia: a systematic scoping review. Alzheimers Res Ther 11:1-11

78. Chiva-Blanch G, Badimon L (2020) Benefits and risks of moderate alcohol consumption on cardiovascular disease: current findings and controversies. Nutrients 12:108

79. Anstey KJ, Mack HA, Cherbuin N (2009) Alcohol consumption as a risk factor for dementia and cognitive decline: meta-analysis of prospective studies. Am J Geriatr psychiatry 17:542-555

80. Schwarzinger M, Thiébaut SP, Baillot S et al (2018) Alcohol use disorders and associated chronic disease-a national retrospective cohort study from France. BMC Public Health 18:43

81. Kuh D, Ben-Shlomo Y, Lynch J et al (2003) Life course epidemiology. J Epidemiol Community Health 57:778-783. https://doi. org/10.1136/jech.57.10.778

82. Kivimäki M, Luukkonen R, Batty GD et al (2018) Body mass index and risk of dementia: Analysis of individual-level data from 1.3 million individuals. Alzheimer's Dement 14:601-609. https:// doi.org/10.1016/j.jalz.2017.09.016

83. Yoo HS, Chung SJ, Lee PH et al (2019) The influence of body mass index at diagnosis on cognitive decline in parkinson's disease. J Clin Neurol 15:517. https://doi.org/10.3988/ jen.2019.15.4.517

84. Sun Z, Wang ZT, Sun FR et al (2020) Late-life obesity is a protective factor for prodromal Alzheimer's disease: a longitudinal study. Aging 12:2005-2017. https://doi.org/10.18632/aging.10273 8

85. Dahl AK, Löppönen M, Isoaho R et al (2008) Overweight and Obesity in Old Age Are Not Associated with Greater Dementia Risk: (See editorial comments by Dr. David S. Knodman, pp 2349-2350). J Am Geriatr Soc 56:2261-2266

86. Kim Y, Suh YK, Choi H (2004) BMI and metabolic disorders in south korean adults: 1998 korea national health and nutrition survey. Obes Res 12:445-453. https://doi.org/10.1038/oby.2004.50

87. Alhurani RE, Vassilaki M, Aakre JA et al (2016) Decline in weight and incident mild cognitive impairment: mayo clinic study of aging. JAMA Neurol 73:439-446

88. Klatsky AL, Zhang J, Udaltsova N et al (2017) Body mass index and mortality in a very large cohort: is it really healthier to be overweight? Perm J. https://doi.org/10.7812/TPP/16-142 
89. Boutari C, Mantzoros CS (2017) Decreasing lean body mass with age: challenges and opportunities for novel therapies. Endocrinol Metab 32:422-425

90. van Loenhoud AC, van der Flier WM, Wink AM et al (2019) Cognitive reserve and clinical progression in Alzheimer disease: a paradoxical relationship. Neurology 93:e334-e346
Publisher's Note Springer Nature remains neutral with regard to jurisdictional claims in published maps and institutional affiliations. 